\title{
Post HCV Infection Due to MX Gene Stimulation Produced Post Treatment with Imported and Locally Produced Egyptian Biosimilar IFN
}

\author{
Shereen H Mohamed ${ }^{1 \& *}$, Nora F Mahmoud ${ }^{2}$, Aly F Mohamed ${ }^{3 \&}$, Nahla S Kotb ${ }^{1}$
}

\begin{abstract}
Background: Cirrhosis is regarded as a possible end stage of many liver diseases, including viral infection. It occurs when healthy liver tissue becomes damaged and is replaced by scar tissue and finally may lead to hepatocellular carcinoma. Interferons (IFNs)are two general categories, type I and II. Type I includes one beta interferon and over 20 different alpha interferons. Alpha interferons are very similar in how they work, interacting with other proteins on cells like receptors. The main objective of this study was to compare Mx gene productivity post different cell line treatment with imported and Egyptian biosimilar locally produced IFNs, as well as the efficacy of those tested IFNs. Also, an assessment was made of sensitivity of different cell lines as alternatives to that recommended for evaluation of antiviral activity. Materials and Methods: Different cell lines (Vero, MDBK and Wish) were employed to evaluate cytotoxicity using the MTT assay. Antiviral activity was evaluated compared with standard IFN against VSV, Indiana strain -156, on tested rh-IFNs (imported; innovated and Egyptian biosimilar locally produced IFNs) in the pre-treated cell lines previously mentioned. The virus was propagated in the Wish cell line as recommended. Finally we estimated up-regulation of the Mx gene as a biomarker. Results: Data recorded revealed that test IFNs were safe in test cell lines. Viability was around $100 \%$. Locally tested interferon did not realize the international potency limits, while the imported one was accepted compared with the standard IFN. These results were the same either using infectivity titer reduction assay or crystal violet staining of residual non- infected cells. Mx protein production was cell type related and confirmed by the detected Mx gene expressed in imported and locally produced IFN pre-treated cell lines. The expression of the gene was arranged in the order of Vero $>$ wish $>$ MDBK for the imported IFN, while for the Egyptian biosimillar locally produced one it was MDBK $>$ Vero $>$ wish. With regard to the antiviral activity there was a significant difference of imported IFN potency compared with the locally produced IFN $(P<\mathbf{0 . 0 5})$, the IFN potential (antiviral activity) was not cell line related and showed non-significant difference for each separate product. Conclusions: Vero cells can be used as an alternative cell line for evaluation of IFN potency in case of unavailable USP recommended cell lines. Alternative potency evaluation assay could be used and proved significant difference in IFN potency in case of local and imported agents. Evaluation of antiviral activity could be used in parallel to viral infectivity reduction assay for better accuracy. Mx gene can be used as a marker for IFN potential.
\end{abstract}

Keywords: Interferon - cell lines - cytotoxicity - Mx gene - antiviral activity

Asian Pac J Cancer Prev, 16 (14), 5635-5641

\section{Introduction}

Interferons (IFNs) are proteins made and released by lymphocytes in response to the presence of pathogens, such as viruses, bacteria, parasites or tumor cells. They allow communication between cells to trigger the protective defenses of the immune system that eliminate pathogens or tumors. IFNs belong to the large class of glycoproteins known as cytokines. Interferons are named after their ability to "interfere" with viral replication cycles within host cells. IFNs have other functions: they activate immune cells, such as natural killer (NK) cells and macrophages; they increase recognition of infectious agents by up-regulating antigen presentation to T lymphocytes; and they increase the ability of uninfected host cells to resist new viral infection. Certain host symptoms, such as aching muscles and fever, are related to the production of IFNs during infection. About ten distinct IFNs have been identified in mammals; seven of these have been described for humans. They are typically divided IFN into 3types (I, II and III IFN), (de Weerd et al., 2007) .

${ }^{1}$ National Organization for Research and Control of Biological (NORCB), Dokki, Giza, ${ }^{2}$ Faculty of Pharmacy, Suez Canal University, Ismailia, Egypt, ${ }^{3}$ The Egyptian Company for production of vaccines, Sera and Drugs (Holding Company (Egy-VAC-VACSERA), Dokki, Giza, Egypt \&Equal contributors*For correspondence: phshereenhamdy@gmail.com 
According to the WHO: A biotherapeutic product which is similar in terms of quality, safety and efficacy to an already licensed reference biotherapeutic product, (World Health Organization, 2009). The active substance of a biosimilar medicine is similar to the one of the biological reference medicine (innovator). Biosimilar and biological reference medicines are used in general at the same dose to treat the same disease as they are intended to have the same mechanism of action, EMEA (2008). Biosimillar and biological reference medicines are similar but not identical regarding something as: the name, appearance and packaging of a biosimillar medicine. It may also contain different inactive ingredients, EMEA (2008). The quality attributes in the similar biological and reference medicinal products are not identical, EMEA (2006). The development of biosimillar based on the comparability to a reference biological product (originator biopharmaceutical). In fact, the legal term introduced during 2003 in the EU Directive 2003/63/EC (amending 2001/83/EC) is "similar biological medicinal product", although such products are commonly known as "biosimilars" (Blanchard et al., 2010; Nick, 2012). The antiviral activity of any antiviral compound is based on its ability to inhibit virus inducing cellular cytopathic effects (CPE) in mammalian cells.

According to the EUROPEAN PHARMACOPOEIA 5.0 regarding INF assay 5.6, the cell lines used in this study were verified to enhance the possibility to use available cell line (Vero) during the evaluation assay to evaluate the IFN potential, despite the pharmacopeial model to perform the antiviral activity assay was to use either Wish or MDBK cell lines. While, vesicular stomatitis virus (VSV) was chosen as viral model according to the EU Pharmacopeia 5.0, 2005 for performing the antiviral assay.

In our study both Egyptian locally produced (biosimilar) and imported IFN (innovator) their active ingredient are interferon alpha $2 \mathrm{a}$ and they were registered for the same indication with the same dose.

Chronic liver disease causes can be any condition that results in the gradual degradation and renewal of the tissue cells with a body's liver. This process usually results in fibrosis or cirrhosis and can be potentially fatal in cases of chronic liver failure. Hepatitis $\mathrm{C}$ progression to cirrhosis is highly variable, depending on the presence of cofactors capable of accelerating the fibrotic process, (EASL, 2014).

Hepatocellular carcinoma (HCC) is one of the most common cancer-related death worldwide (Parkin et al., 2001; Xiao-Chun et al., 2014). Early intervention is critical to obtain survival benefit. Therefore reliable markers to identify patients who are at high risk for early death and recurrence would be necessary. Based on the biomarkers potentials for diagnosis and control of viral infection and related HCC the present work aims to minimize the chances for incidence of liver cancer induction due to $\mathrm{HCV}$ infection that detected based on the bioactivity of IFN to inhibit virus replication in liver tissues via induction of $\mathrm{Mx}$ protein producing gene expression in interferon treated cell lines under the effect of both innovator and locally produced IFN. Also evaluation of Vero as a virus replication host cell sensitivity to detection of antiviral activity potential compared with those recommended by the EU-pharmacopeia namely MDBK and WISH cells.

\section{Materials and Methods}

\section{Virus model and cell lines}

Vesicular stomatitis virus (VSV), Indiana strain-156 was kindly supplied by Biotechnology Unit, NORCBEgypt. Virus infectivity titer was determined according to reported method, (Steven et al., 2009), the VSV was 10 fold serially diluted. Each dilution was dispensed as $100 \mu \mathrm{L} /$ well onto pre-culture Vero cells. Non infected wells as negative cell control were considered. Plates were incubated at $37^{\circ} \mathrm{C} .7$ days post infection the $50 \%$ end point (CCID50) was determined by the method of (Reed and Muench, 1938).

In the present study, three cell lines were used, namely Vero CCL-81, Wish, CCL - 25 and MDBK, CCL - 22 were kindly supplied from Cell Culture Department, VACSERA -Egypt. Cells were grown in 199-E and D-MEM supplied from (GIBCO-UK). Growth medium was supplemented with $10 \%$ inactivated fetal calf serum (FCS), $5 \mathrm{mM}$ HEPES buffer and antibiotics (100U of penicillin/ml, $100 \mathrm{gm}$ of streptomycin $/ \mathrm{ml}$ ) at $37^{\circ} \mathrm{C}$ and incubated in $5 \% \mathrm{CO}_{2}$ atmospheric conditions (Jouan-France).

\section{Interferon}

Innovator imported interferon samples and Egyptian locally produced biosimilar one were used in this study to compare their potency as free interferon. Both imported (innovator) interferon and Egyptian locally produced (non-innovator / biosimilar) are free (non-pegylated) alpha 2 a. IFN was 10 fold serially diluted in MEM-Earl's (GIBCO- UK) supplemented with $10 \%$ fetal calf serum (GIBCO- UK).

\section{Reference standard}

The reference IFN used in the antiviral assay was "WHO International Standard INTERFERON ALPHA 2a, (HUMAN, rDNA derived) NIBSC code: 95/650).

\section{Antiviral activity}

Antiviral activity of Interferons, against VSV was determined using two different techniques: the 1st one was according to the method described in the European Pharmacopoeia (5.0) INF assays (5.6.), as a standard protocol for IFN potency evaluation, where IFN used as $5000 \mathrm{IU} / \mathrm{ml}$ was twofold serially diluted and used for the treatment of pre-cultured cell lines as $0.1 \mathrm{ml} /$ well. The VSV was used as (1000 TCID50) in 199 E-Hepes buffered. Antiviral activity was determined by determination of the highest dilution of IFN that could inhibit the used VSV infectivity titer recommended by the European Pharmacopeia.

\section{Alternative antiviral activity}

The alternative antiviral assay was performed according to (Shinji, 2005, Samir et al., 2013), where antiviral activity of test rh-IFNs was determined by subtracting the virus infectivity titer recorded in case of $10 \mathrm{IU} / 1 \mathrm{ml} 24 \mathrm{hrs}$ pretreated cultured cells from virus infectivity titer of IFN non treated cells. The difference 
Post HCV Infection Due to MX Gene Stimulation with Imported and Locally Produced Egyptian Biosimilar IFNs

represents the antiviral activity. One plate was left untreated for viral control titration. Virus infectivity titer was determined according to a reported method of (Steven et al., 2009), where VSV was tenfold serially diluted in hanks balanced salt solution (10-1-10-7). Each dilution was dispensed as $100 \mu \mathrm{l} /$ well onto pre-cultured test cell lines. Plate was incubated at $37 \mathrm{oC}$. Seven days post infection the $50 \%$ end point (CCID50) was determined according to the method of (Reed and Muench, 1938).

\section{Primer selection}

A highly conserved region found among several mammalian Mx nucleotide sequences (400bp) of the Mx gene was selected. This region was found in EMBL, GenBank and DDBJ nucleotide sequence data bases under the following accession numbers (NM002462, BC032602, AF047692, and NM204609). The primer designer computer software program (DNA star programs) was used to select primers of interest. The forward primer $(\mathrm{F})$ sequence was 5' CTGCCCCTGTTAGAAAATCAAATC $3^{\prime}$, and the reverse primer $(\mathrm{R})$ sequence was 5' ATATCCACAGCCGGCTCTTCCAGT 3'.

\section{Total RNA extraction}

Vero, Wish and MDBK cell lines were cultured in tissue culture flask $25 \mathrm{ml}$ total volume. Tissue culture flasks were treated with IFN using under test. IFN treated media were decanted from the flasks $24 \mathrm{hrs}$. post treatment. Cells were treated with trypsin to dissociate treated cells from the surface of the culture flask then cold centrifuged (Jouan KR France 41 ) for $15 \mathrm{~min}$ and $+4^{\circ} \mathrm{C}$ to proceed for RNA extraction.

\section{Reverse transcription and polymerase chain reaction}

DNA double strand was synthesized using one tube RT-PCR system (Roche Applied Science, Penzberg, Germany). In a single vial, two stage protocols, RT and PCR reagents were combined in a single mixture. The first strands cDNAs synthesis was accomplished by incubation at $50^{\circ} \mathrm{C}$ for 30 minutes, then the amplification conditions were started with 5 minutes at $94^{\circ} \mathrm{C}$ followed by 35 cycles of $94^{\circ} \mathrm{C}$ for 60 seconds; $60^{\circ} \mathrm{C}$ for 45 seconds; $72^{\circ} \mathrm{C}$ for 60 seconds. The PCR products were finally analyzed on $1 \%$ agarose gel electrophoresis (Sambrook et al., 1989).

\section{Statistical analysis}

Statistical analysis was performed using the analysis of variance (ANOVA) to determine the difference between treated and control samples (mean \pm S.D were calculated), the $\mathrm{P}$ value was $<0.05$. All statistics were carried out using Statistical Package for Social Science (SPSS, 1999 program).

\section{Results}

Cytotoxicity assay performed revealed that all test IFNs were safe to different cell lines used as viability $\%$ was around $100 \%$. [Figure1]. Regarding to antiviral activity data recorded revealed that the concentration that could inhibit the recommended challenge dose of VSV (1000 TCID50) was 51, 54 and $59.6 \mathrm{IU} / \mathrm{ml}$ for imported IFN and 260, 263.4 and 256 for Egyptian locally produced one when tested on Vero, Wish and MDBK respectively [Figure 2], and there was a noticed a significant difference $(\mathrm{P}<0.05)$ of imported IFN potentials compared to Egyptian locally produced one [Figure 3]. As well as the antiviral activity recorded values revealed that the difference between the different cell lines used for the same product was statistically insignificant $(\mathrm{P}>0.05)$, thus we accept the null hypothesis [Figure 4]. In the same way an alternative protocol for evaluation of antiviral activity was performed, and data revealed that the log difference of virus infectivity titer determined post cell treatment with imported IFN was significantly higher than that induced post cell treatment with Egyptian local IFN compared to imported one $(\mathrm{P}<0.05)$, [Figure 5]. In the mean time detection of Mx - mRNA as a biomarker was performed and was detected in variable \% relatively to the cell line

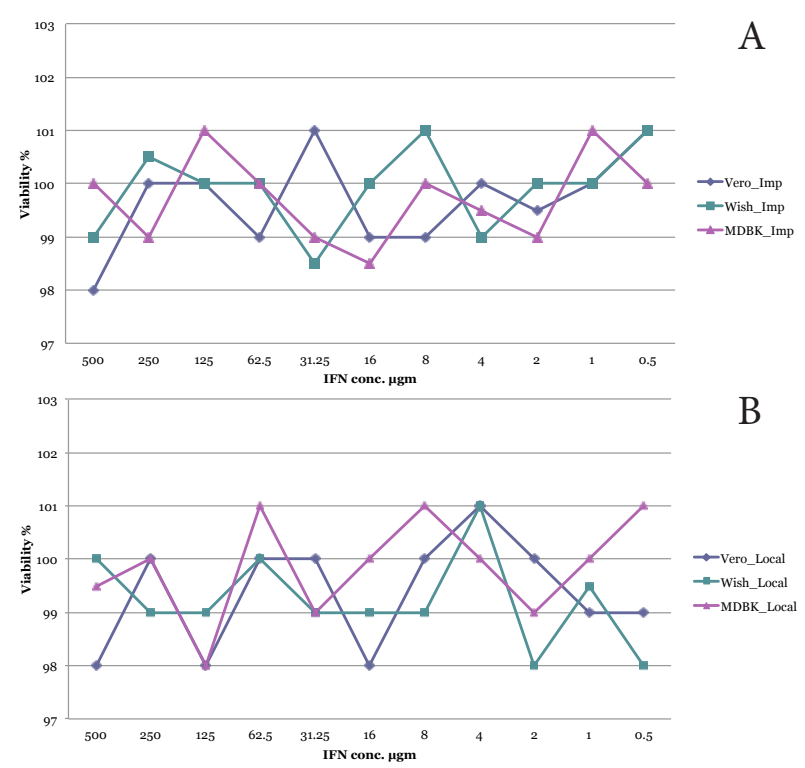

Figure 1. Evaluation of Cytotoxicity of Interferon.

Cytotoxicity assay performed revealed that all test IFNs were safe to different cell lines representing the viability \% that was around $100 \%$, A) Evaluation of cytotoxicity of interferon for Imported IFN, B) Evaluation of cytotoxicity of interferon for local IFN

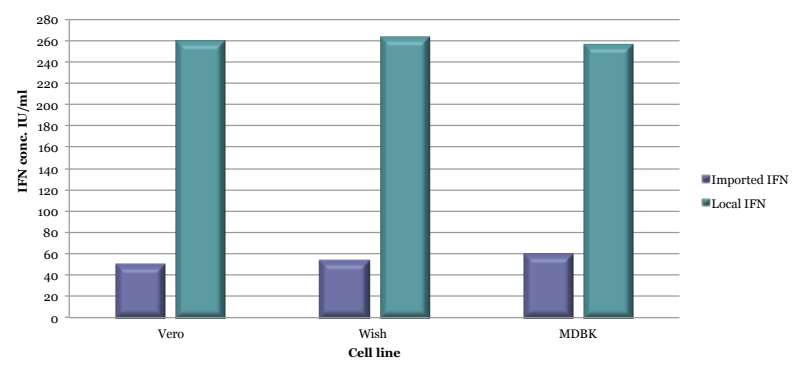

Figure 2. Assessment of Inhibitory Concentration of Egyptian Locally Produced and Imported Interferons Used Against VSV TCID50. This figure describes the concentration of both IFN under test that could inhibit the recommended infectivity titer (IT) of VSV ;( 1000 TCID50). The results was 51,54 and $59.6 \mathrm{IU} / \mathrm{ml}$ for imported IFN and 260, 263.4 and 256 for Egyptian locally produced one when tested on Vero, Wish and MDBK cell lines respectively 


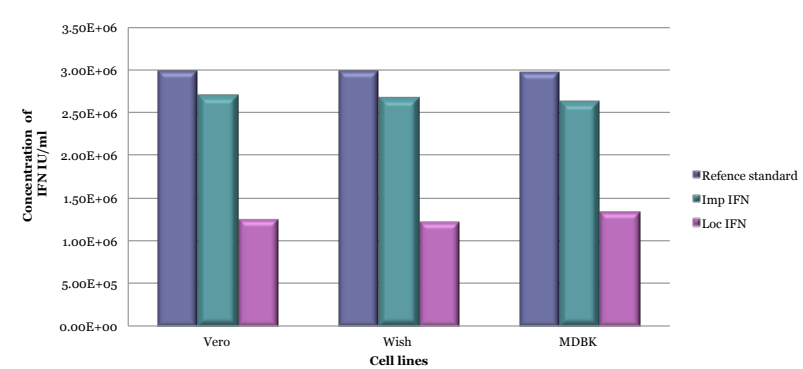

Figure 3. Comparative Evaluation of Antiviral Activity of Imported and Egyptian Local Interferons Regarding the International Standards. Regarding the international standards reference used to evaluate the antiviral activity of both Egyptian locally produced and imported IFNs and regarding to the acceptance limit according to pharmacopeia; $80 \%-125 \%$ of the main concentration $(3.00 \mathrm{E}+06 \mathrm{IU} / \mathrm{ml})$ i.e $2.40 \mathrm{E}+06$ $-3.75 \mathrm{E}+06$ the resulted data revealed significant difference $(\mathrm{P}<0.05)$ of imported IFN potentials compared to the Egyptian locally produced one as well as the imported IFN showed accepted results rather than the locally produced biosimillar

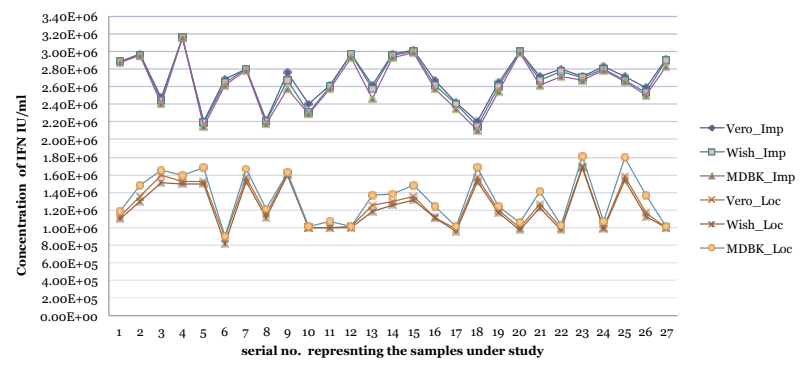

Figure 4. Comparative Evaluation of Antiviral Activity of Imported and Locally Produced Interferons Using Different Cell Lines. By comparing the antiviral activity of each of both the imported (innovator) and the Egyptian locally produced (biosimillar) using different cell lines which were mentioned in the pharmacopeia (Wish and MDBK) for the antiviral assay in addition to another available one (Vero cell line) the recorded results revealed that the difference between the three cell lines used for the same product was statistically insignificant $(\mathrm{P}>0.05)$, thus any of these cell lines used in this study can be used in evaluating the antiviral activity assay

Table 1. Determination of Mx Gene Expression (Band Intensity) Using Spectrophotometery

\begin{tabular}{ccc}
\hline $\begin{array}{l}\text { Serial No. of } \\
\text { test sample }\end{array}$ & Treatment & $\begin{array}{c}\text { Band } \\
\text { Intensity }\end{array}$ \\
\hline 1 & Wish cells Imported IFN treated & 225 \\
2 & Wish cells Local IFN treated & 144 \\
3 & MDBK cells Imported IFN treated & 178 \\
4 & MDBK cells Local IFN treated & 197 \\
5 & Vero cells Imported IFN treated & 230 \\
6 & Vero cells Local IFN treated & 147 \\
7 & Wish cells negative control & - \\
8 & MDBK cells negative control & - \\
9 & Vero cells negative control & - \\
\hline
\end{tabular}

used, and the Mx gene band intensities [Figure 6] detected in case of imported IFN could arranged in the order of Vero $>$ Wish $>$ MDBK [Table 1]. While in case of testing

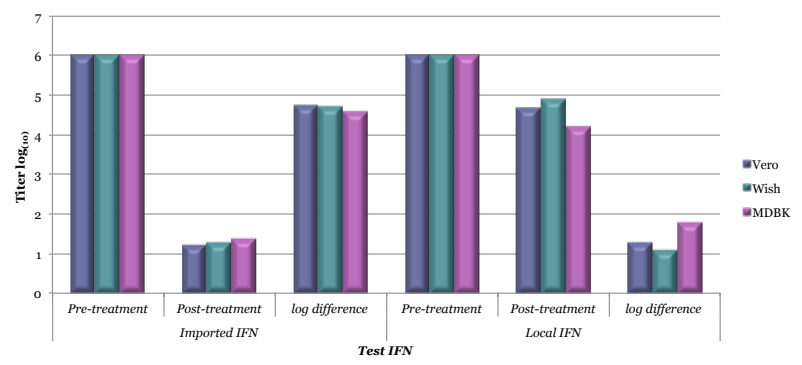

Figure 5. Evaluation of Antiviral Activity of test rhInterferon Using Constant Concentration of rh-IFN. An alternative protocol for evaluation of antiviral activity was performed, as VSV was tenfold serially diluted in hanks balanced salt solution $\left(10^{-1}-10^{-7}\right)$. Each dilution was dispensed as $100 \mu \mathrm{l} /$ well onto pre-cultured test cell lines. Plate was incubated at $37^{\circ} \mathrm{C}$. By subtracting the virus infectivity titer recorded in case of 10 IU/ $1 \mathrm{ml} 24 \mathrm{hrs}$ pretreated cultured cells from virus infectivity titer of IFN non treated cells. The difference represents the antiviral activity. These data revealed that the log difference of virus infectivity titer determined post cell treatment with imported IFN was significantly higher than that induced post cell treatment with Egyptian locally produced IFN compared to imported one $(\mathrm{P}<0.05)$.

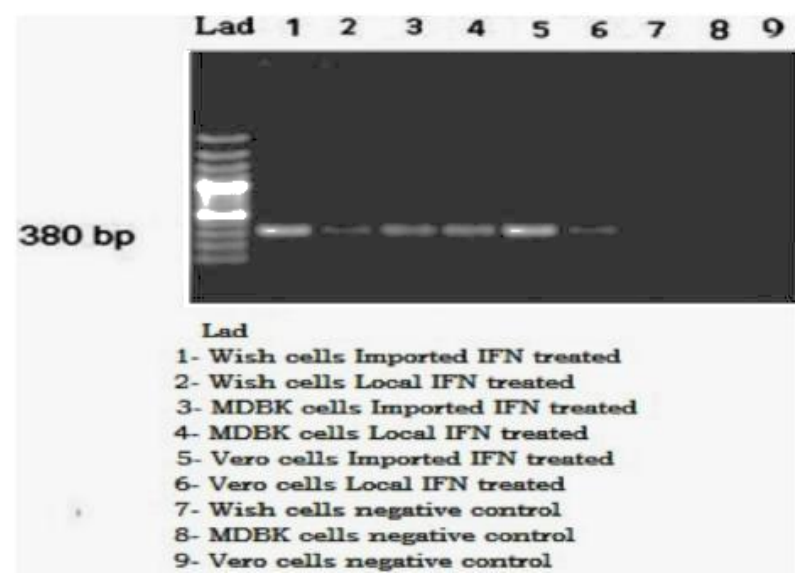

Figure 6. Detection of Mx Gene Post IFN Treatment Detection of Mx-mRNA as a biomarker was performed and was detected in variable \% relatively to the cell line used, and the Mx gene band intensities [Figure 6] detected in case of imported IFN could arranged in the order of Vero $>$ Wish $>$ MDBK [Table 1]. While in case of testing the Egyptian biosimillar (Local IFN) gene band was of lesser intensity [Table 1] and arranged in the order of MDBK $>$ Vero $>$ Wish

the biosimillar (Egyptian Local IFN) gene band was of lesser intensity [Table 1] and arranged in the order of MDBK $>$ Vero $>$ Wish.

\section{Discussion}

The fact that the majority of HCC patients have an underlying chronic inflammatory liver disease and liver cirrhosis is the main risk factor for $\mathrm{HCC}$ development (Gao et al., 2012). Chronic hepatitis C patients cumulative risk for HCC was evaluated according to the use of IFN therapy and their response to the therapy. HCC risk was very high among the patients without IFN treatment or 
those who didn't respond to IFN therapy. In contrast, responders to IFN remained at minimal to moderate risk for HCC, according to the duration of response. A sustained response, with patients remaining at minimal risk for $\mathrm{HCC}$, means that transaminases normalization continued over 6 months after completing IFN therapy. As compared to the risk among patients without IFN treatment, the multivariate-adjusted hazard rate ratio for HCC was 0.52 for those who received IFN therapy (Hideaki et al., 2005).

Production of interferons predominantly occurs in response to microbial exposure; viruses and bacteria, and toxins. Binding of molecules uniquely found in microbes, viral glycoproteins, viral RNA, bacterial endotoxin (lipopolysaccharide), bacterial flagella, $\mathrm{CpG}$ motifs by pattern recognition receptors, such as membrane bound Toll like receptors or the cytoplasmic receptors RIG-I or MDA5, can trigger release of IFNs. Tremendous progress has been made in understanding the molecular basis of the antiviral actions of interferons (IFNs), as well as strategies evolved by viruses to antagonize the actions of IFNs. Furthermore, advances made while elucidating the IFN system have contributed significantly in multiple areas of virology and molecular cell biology, ranging from pathways of signal transduction to the biochemical mechanisms of transcriptional and translational control to the molecular basis of viral pathogenesis.

IFNs are approved therapeutics and have moved from the basic research laboratory to the clinic. The availability of IFN-free treatment regimens has simplified hepatitis C therapy with shorter-duration, all-oral therapy for many patients (AASLD).

Several additional genes whose expression profiles are altered in response to IFN treatment and virus infection have been identified by microarray analyses, (Mehta et al., 2012).

Among the IFN-induced proteins important in the antiviral actions of IFNs are the RNA-dependent protein kinase (PKR), the 2', 5'-oligoadenylate synthetase (OAS) and RNase L, and the Mx protein GTPases. The latter is a protein that is encoded by the MX1 gene in human, (Haller et al., 2007; Entrez Gene, 2013). While, in mouse, the interferon-inducible $\mathrm{Mx}$ protein is responsible for a specific antiviral state against influenza virus infection. And the protein encoded by this gene is similar to the mouse protein as determined by its antigenic relatedness, induction conditions, physicochemical properties, and amino acid analysis. This cytoplasmic protein is a member of both the dynamin family and the family of large GTPases, (Horisberger, 1992; Pavlovic et al., 1992). Regarding the antiviral activity, data recorded proved that the double-stranded RNA plays a central role in modulating protein phosphorylation and RNA degradation catalyzed by the IFN-inducible PKR kinase and the 2'-5'-oligoadenylate-dependent RNase L, respectively, and also in RNA editing by the IFN-inducible RNAspecific adenosine deaminase (ADAR1).

Use of IFN as antiviral therapy, in both cell culture and animals, continues to provide important contributions to our understanding of the virus-host interaction and cellular antiviral response. In the present study, antiviral activity of innovator and biosimilar evaluated using two different assessment procedures. Data revealed that innovator IFN potency was significantly more effective than biosimillar, these data was in accordance with (Manns et al., 2001; Ge et al., 2009) despite their use of modification achieved by using Ribaverin as enhancer to PEGIFN and Standard biosimillar IFN, the former was more effective than the biosimillar standard IFN. And these data was in accordance to ours, although our used model was non pegilated IFN (free) as no pegilated biosimillar available. Also, these obligations were enhanced by (Aline et al., 2012) that Biosimillar of Peg-IFN is not available, and the only Peg-IFN formulations currently available are Peg-IFN alpha-2a or alpha-2b.

Antiviral activity variability may attributed to the IFN behavior that still completely unclear and it may be related to the production and transfection protocols. Regarding the inhibitory effect of IFN to HCC was approved by (Hiteaki et al 2005) that IFN could be used with or without Ribavirine to be effective for reducing the risk of HCC among patients with chronic Hepatitis $\mathrm{C}$.

Also, antiviral occurrence may be attributed to viral coat synthesis inhibition and to the production of nitric oxide (NO) which known to have antiviral activity, K D (1993), as nitric oxide is a chemical compound with pleiotropic functions; its production by phagocytes in response to interferon- $\gamma$ is associated with antimicrobial activity; as in response to dsRNA, nitric oxide is rapidly produced in primary fibroblasts. In the presence of an intact interferon system, nitric oxide plays a minor but significant role in antiviral protection. In primary fibroblasts, NF- $x \mathrm{~B}$ and interferon regulatory factor 1 participate in the induction of inducible nitric oxide synthase expression, which subsequently produces nitric oxide. Inducible nitric oxide synthase (iNOS2) and the major histocompatibility complex class I and II proteins, all of which play important roles in immune response to infections.

Regarding the application of IFN; use of 10 IU was according to the recommendations of the producers and E. pharmacopeia. This concentration and related results were in agreement to the report of (JAMES et al., 1998) recording that IFN activity can be measured accurately over a concentration range of 0.1-30 IU/ml. Also, they recorded that the $\mathrm{MxA}$-induction assay has advantages in terms of specificity, reliability, and sensitivity over other methods for assaying type I IFN.

The role of $\mathrm{Mx}$ as a biomarker traced in our present study that was detectable in different cell lines used and the intensity of expression of Mx gene was related to the interferons either innovator or biosimillar and cell lines used, this was in accordance to (Mohamed et al., 2006) were the Mx protein has successfully been used as a marker in the cells treated with rh-IFN- $\alpha$ and Mx gene expression was sufficient as a bio- marker.

The genomic nucleic acids serve as molecular signatures associated with viral infections. It was found that TLR7 recognizes the single-stranded RNA viruses, vesicular stomatitis virus and influenza virus. The recognition of these viruses by plasmacytoid dendritic cells and B cells through TLR7 results in their activation 
of co-stimulatory molecules and production of cytokines. Moreover, this recognition required intact endocytic pathways. Mice deficient in either the TLR7 or the TLR adaptor protein MyD88 demonstrated reduced responses to in vivo infection with vesicular stomatitis virus. These results demonstrate microbial ligand recognition by TLR7 and provide insights into the pathways used by the innate immune cells in the recognition of viral pathogens, (Jennifer et al., 2004).

Also, our data was in accordance to that recorded by (Doris et al., 2002) despite his trials were on ds-RNA viruses recording those IFN-regulated genes, such as the ones for double-stranded-RNA-dependent protein kinase, $2^{\prime}-5^{\prime}$-oligoadenylate synthetase, or the Mx proteins. In (Doris et al., 2002) study, cultured MDBK cells and bovine white blood cells (WBC) were treated with recombinant IFN- $\alpha$ or infected with either bovine herpes virus 1 (BHV1) or bovine rotavirus (BRV). Treatment of cultured cells with IFN- $\alpha$ was followed within four hours by a time- and dose-dependent accumulation of intra-cytoplasmic $\mathrm{Mx}$ protein as revealed by immunostaining and Western blot immunoassay. This was preceded by a distinct rise of $\mathrm{Mx}$ mRNA in similarly treated cells, as revealed by a newly established quantitative TaqMan PCR technique.

The antiviral potential of rh-IFN- $\alpha$ was attributed to the general inhibition activity of viral protein synthesis or transcription (Baglioni and Nilsen, 1984; Korth et al., 1998). MxA, rh-IFN- $\alpha$ detection, has been reported to inhibit replication of certain RNA viruses (Ponten et al., 1997; Abel et al., 2002). Rh-IFN- $\alpha$ treated cells were subjected to RT-PCR to clarify whether or not the enhancement of such MxA expression is mediated by rh-IFN- $\alpha$. Also inducible up regulation of Mx gene was proved by (Kenneth and Worthen, 2003) under the effect of rh-IFN- $\alpha$ even if the cells were significantly resistant to viral infection. (Sahapat et al., 2014) regarded the effect of IFN as cancer therapy, our study aimed to monitoring the antiviral activity of both imported and biosimillar locally produced IFNs which was confirmed by detection of Mx protein as a biomarker that can enhance the reduction of viral load in turn can minimize the incidence of HCC.

Regarding Mx gene, it was proved that there are a panel of genes can be used as biomarkers as (Xiao-Chun et al., 2014) used serum amyloid A (SAA) as a biomarker for prognosis of HCC patients.

Finally it can be concluded that antiviral activity can be accurately conducted using a wide range of alternative cell lines than those recommended by the producers and pharmacopeias. Mx gene as a biomarker can be concerned as an indication for the antiviral activity potentials and Mx gene expression was sufficient sensitive biomarker to abolish the resistance phenotype to viral infection in case of hepatic patients. Also, innovator IFN product showed better potential than target Egyptian biosimillar. In addition, local evaluation of IFN potentials could be established using different cell lines in the National Regulatory Authority [NRA] in Egypt efficiently compared to the international protocols.

Recommendations: 1-Different cell line can be used efficiently in parallel to the manufacturer recommended cell line; 2- Variable virus models could be tried instead of VSV as some are used; Bovine virus diarrhea (BVD) and Encephalomyocarditis virus (EMV) to validate the optimum antiviral activity relative to different virus models; 3-More detailed investigations for the cell culture Mx gene, sequences and expression rate Traceability using Real time- PCR for more accurate results than confidential PCR; 4- Antioxidant profile should be monitored trying to find out if it may have a role in antiviral potentials.

\section{Acknowledgements}

The authors gratefully thank Prof Dr. Sayed Salim for his recommendations and his great efforts. Thanks to VACSERA R\&D sector and NORCB for facilitating the laboratory technical and financial supports.

\section{References}

Abel K, Algeria-Hartman MJ, Rothaeusler K, Marthas M, Miller CJ (2002). The relationship between Simian Immunodeficiency virus RNA levels and the mRNA levels of Alpha/Beta Interferon (IFN $\alpha / \beta$ ) and IFN $\alpha / \beta$-inducible MX in Lymphoid tissue of Rhesus Macaques during Acute and Chronic infection. $J$ Virol, 76, 8433-45.

Aline Gonzalez Vigani, Eduardo Sellan Goncales, Maria Helena Postal Pavan, et al (2012). Therapeutic effectiveness of biosimilar standard interferon versus pegylated interferon for chronic hepatitis C genotypes 2 or 3. Braz J Infect Dis, 16, 232-6.

AASLD (American Association for the Study of Liver Diseases). Recommendations for testing, managing and treating hepatitis C. Monitoring patients who are starting hepatitis C treatment, are on treatment, or have completed therapy http:// www.hcvguidelines.org/full-report/monitoring-patientswho-are-starting-hepatitis-c-treatment-are-treatment-orhave

Baglioni C, Nilsen TW (1984). in Gresser 1 (ed) Interferon. Vol.5. Academic Press. New York p23; 1984.

Blanchard A, Helene D'Iorio and Robert Ford (2010). What you need to know to succeed: Key trends in Canada's biotech industry. Insights, spring.

Doris Müller-Doblies, Mathias Ackermann and Alfred Metzler (2002). In vitro and in vivo detection of $\mathrm{mx}$ gene products in bovine cells following stimulation with alpha/beta interferon and viruses. Clinical Vaccine Immunol, 9, 1192-9.

de Weerd NA, Samarajiwa SA and Hertzog PJ (2007). Type I interferon receptors: biochemistry and biological functions. J Biol Chem, 282, 20053-7.

EMEA (2006). Guideline on similar biological medicinal products containing biotecnology-derived proteins as active substance: QUALITY ISSUES.

EMEA (2008). D.R.E.R., Questions and answers on biosimilar medicines (similar biological medicinal products). London.

Entrez Gene (2013). MX1 myxovirus (influenza virus) resistance 1, interferon-inducible protein $\mathrm{p} 78$ (mouse).

EASL (European Association for the Study of the Liver) (2014). Clinical practice guidelines: management of hepatitis $\mathrm{C}$ virus infection. J Hepatol, 60, 392-420.

Ge D, Fellay J, Thompson AJ, et al (2009). Genetic variation in IL28B predicts hepatitis $C$ treatment-induced viral clearance. Nature, 461, 399-401.

Gao J, Xie L, Yang WS, et al (2012). Risk factors of hepatocellular carcinoma--current status and perspectives. Asian Pac J Cancer Prev, 13, 743-52.

Horisberger MA (1992). Interferon-induced human protein MxA is a GTPase which binds transiently to cellular proteins. $J$ 

Virol, 66, 4705-9.

Haller O, Staeheli P, Kochs G (2007). Interferon-induced Mx proteins in antiviral host defense. Biochimie, 89, 812-8.

Hideaki Tsukuma, Hideo Tanaka, Wakiko Ajiki, Akira Oshima (2005). Liver cancer and its prevention. Asian Pac J Cancer Prev, 6, 244-50.

James G. Files, Julia L. Gray, Linh T. Do, et al (1998). A novel sensitive and selective bioassay for human type i interferons. J Interferon Cytokine Res, 18, 1019-24.

Lund JM1, Alexopoulou L, Sato A, et al (2004). Recognition of single-stranded RNA viruses by Toll-like receptor 7. Proc Natl Acad Sci U S A, 101, 5598-603.

KD Croen (1993). Evidence for antiviral effect of nitric oxide. Inhibition of herpes simplex virus type 1 replication. J Clin Invest, 91, 2446-52.

Korth M.J, Taylor M.D, Katze M.G (1998). Interferon inhibits the replication of HIV-1, SIV and SHIV chimeric viruses by distinct mechanisms. Virology, 247, 265-73.

Kenneth C.M, Worthen S (2003). Lipopolysaccharide stimulates p38-dependent induction of antiviral genes in neutrophils independently of paracrine factors. J Biol Chem, 278, 15693-701.

Manns MP, McHutchison JG, Gordon SC, et al (2001). Peginterferon alfa- $2 \mathrm{~b}$ plus ribavirin compared with interferon alfa- $2 b$ plus ribavirin for initial treatment of chronic hepatitis C. Randomised trial. Lancet, 358, 958-65.

Mohamed A.F, Shoman S.A (2006). Antiviral activity of recombinant human interferon- $\alpha$ to rift valley fever virus and related Mx-protein potential as a biomarker. Egy $J$ Biotechnol, 21, 203-6.

Mehta DR, Ashkar AA, Mossman KL (2012). The nitric oxide pathway provides innate antiviral protection in conjunction with the type i interferon pathway in fibroblasts. PLOSONE, 7, 31688 .

Nick C (2012). The US biosimilars act: challenges facing regulatory approval. Pharm Med, 26, 2145-52.

Pavlovic J, Haller O, Staeheli P (1992). Human and mouse Mx proteins inhibit different steps of the influenza virus multiplication cycle. J Virol, 66, 2564-9.

Ponten A, Sick C, Weeber M, Haller O, Kochs G (1997). Dominant-negative mutants of human MXA protein: domains in the carboxy-terminal moiety are important for oligomerization and antiviral activity. $J$ Virol, 71, 2591- 9.

Parkin DM, Bray F, Ferlay J, Pisani P (2001). Estimating the world cancer burden: Globocan 2000. Int J Cancer, 94, $153-6$.

Reed LJ, Muench H (1938). A simple method of estimating fifty percent end-points. Am J Hygiene, 27, 493-7.

Sambrook J, Fritch E.F, Maniatis T (1989). Molecular cloning: A Laboratory Manual Cold Spring Harbor Laboratory, Cold Spring Harbor, NY.

Shinji H (2005). The broad anti-viral agent glycyrrhizin directly modulates the fluidity of plasma membrane and HIV-1 envelope. Biochem J antiviral, 392, 191-9.

Steven S, Richard LH, Stephen AY, Weidbrauk DL (2009). Clinical virology manual, 4th ed, ASM press, pp 1-692.

Sahapat Barusrux, Chatchawan Sengthong, Yupa Urwijitaroon (2014). Epidemiology of hepatitis C virus genotypes in northeastern Thai blood samples. Asian Pac J Cancer Prev, 15, 8837-42

Samir Y Abbas, Awatef A Farag, Yousry A, Ammar, et al (2013). Synthesis, characterization, and antiviral activity of novel fluorinated isatin derivatives. Monatsh Chem, 144, 1725-33.

World Health Organization (2009). Expert committee on biological standardization: guidelines on evaluation of similar biotherapeutic products (SBPs). World Health Organization. http://www.who.int/biologicals/areas/biological_ therapeutics/BIOTHERAPEUTICS_FOR_ WEB_22APRIL2010.pdf

http://www.who.int/biologicals/publications/trs/areas/ biological_therapeutics/TRS_977_Annex_2.pdf?ua=1

Xiao-Chun Ni, Yong Yi, Yi-Peng Fu, et al (2014). Serum Amyloid A is a Novel Prognostic Biomarker in Hepatocellular Carcinoma. Asian Pac J Cancer Prev, 15, 10713-8. 